\title{
Gestión de la calidad y su incidencia administrativa financiera en las instituciones de Educación Superior
}

\section{Quality management and its financial administrative impact in Higher Education institutions}

\author{
Alba Lucía Cáceres Larreátegui \\ acaceresl@hotmail.com \\ Universidad Laica Eloy Alfaro de Manabi, Manta \\ https://orcid.org/0000-0001-6805-5823 \\ Ecuador \\ Sandra Ximena Torres Mejía \\ Ximenatorresmejia@gmail.com \\ Universidad Laica Eloy Alfaro de Manabi, Manta \\ https://orcid.org/0000-0003-1649-4079 \\ Ecuador \\ Beatriz Jacqueline Briones Arteaga \\ jackybrionesa15@hotmail.com \\ Universidad Laica Eloy Alfaro de Manabi, Manta \\ https://orcid.org/0000-0002-5219-3276 \\ Ecuador
}

Recibido: 1 de junio del 2019

Aprobado: 30 de Junio del 2019

\begin{abstract}
RESUMEN
La actual investigación se abordó desde una metodología correlacional transeccional, con la finalidad de conocer la relación de la gestión de la calidad y su incidencia administrativa financiera en las instituciones de Educación Superior. Entre los principales resultados se tiene que la correlación de Pearson posee un resultado de 0,063 , la cual se encuentra en el rango de correlación positiva media y una significancia de 0,434, acercándose a 0,5, generando un nivel de confianza positiva. Concluyéndose que la educación de calidad engloba diversos factores que deben ser tenidos en cuenta para la conformación de una gestión basada en lo sistémico como alternativa para consolidar una visión organizativa acorde a los retos globales, lo cual implica formar desde lo glocal para el mundo.
\end{abstract}




\title{
CIENCIAMATRIA
}

Revista Interdisciplinaria de Humanidades, Educación, Ciencia y Tecnología

Año VI. Vol. VI. №10. Enero - Julio 2020

Hecho el depósito de ley: pp201602FA4721

ISSN-L: 2542-3029; ISSN: 2610-802X

Universidad Nacional Experimental Francisco de Miranda (UNEFM). Santa Ana de Coro. Venezuela

Alba Lucía Cáceres Larreátegui; Sandra Ximena Torres Mejía; Beatriz Jaqueline Briones Arteaga

Descriptores: Enseñanza pública; Sistema educativo; Instituciones de enseñanza; Administración de la educación; Gestión educacional.

\begin{abstract}
The current research was approached from a translational correlational methodology, in order to know the relationship of quality management and its financial administrative impact in Higher Education institutions. Among the main results, Pearson's correlation has a result of 0.063 , which is in the average positive correlation range and a significance of 0.434 , approaching 0.5 , generating a positive level of confidence. Concluding that quality education encompasses various factors that must be taken into account for the conformation of a systemic-based management as an alternative to consolidate an organizational vision according to global challenges, which implies forming from the glocal to the world.
\end{abstract}

Descriptors: Public education; Educational systems; Educational institutions; Educational administration; Educational management.

\section{INTRODUCCIÓN}

La globalidad implica transitar hacia escenarios sociales integrados y conectados entre sí, donde las instituciones públicas y privadas deben brindar apoyo al desarrollo integral de la sociedad, así en un momento histórico marcado por el enfoque de lo sostenible y sustentable, se hace necesario recurrir a prácticas empresariales que permitan ser corresponsables de un contexto social en calidad, siendo que la educación universitaria juega un multi papel en este sentido, dado que es empresa que actua en la formación de la generación de relevo productiva de la nación.

Esto implica optimizar los escenarios universitarios desde un enfoque basado en la gestión de procesos como elemento de calidad, esto en la medida que se conforma un accionar que permite trabajar en la mejora continua, promoviendo un trabajo paradigmático desde lo dinámico, flexible, donde se percibe la transcendencia del paradigma clásico mecanicista, basado en estimulo - respuesta.

Así las instituciones de educación superior, ejecutan un accionar complejo, en donde todos los factores que la conforman se encuentran conexos con el propósito de vincular escenarios de calidad, entendiéndose esta el compromiso puertas adentro y fuera de la institución, es decir, que cada miembro de la organización trabaja 


\section{CIENCIAMATRIA}

Revista Interdisciplinaria de Humanidades, Educación, Ciencia y Tecnología

Año VI. Vol. VI. №10. Enero - Julio 2020

Hecho el depósito de ley: pp201602FA4721

ISSN-L: 2542-3029; ISSN: 2610-802X

Universidad Nacional Experimental Francisco de Miranda (UNEFM). Santa Ana de Coro. Venezuela

Alba Lucía Cáceres Larreátegui; Sandra Ximena Torres Mejía; Beatriz Jaqueline Briones Arteaga

sinérgicamente en consecución de metas comunes, siendo y una de ellas, la optimización de los recursos administrativos - financieros, por consiguiente en la actual investigación se persigue mediante una investigación correlacional, comprobar o desechar las siguientes hipótesis:

Hipótesis nula HO: la gestión de la calidad no se relaciona con la competencia administrativa financiera en las instituciones de Educación Superior del Ecuador. Hipótesis positiva $\mathrm{H} 1$ : la gestión de la calidad se relaciona con la competencia administrativa financiera en las instituciones de Educación Superior del Ecuador.

\section{REFERENCIAL TEÓRICO}

\section{Gestión de calidad}

La calidad por gestión en las organizaciones, tiene su fundamento en la Norma ISO, definida por la Organización Internacional de Normalización (ISO) (2015):

Este estándar se basa en una serie de principios de gestión de la calidad que incluyen un fuerte enfoque en el cliente, la motivación, la aplicación de la alta dirección, el enfoque del proceso y la mejora continua. El uso de ISO: 90012015 ayuda a garantizar que los clientes obtengan productos y servicios consistentes y de buena calidad, lo que a su vez aporta muchos beneficios comerciales (p.1).

Así busca que las organizaciones mejoren su calidad específicamente sobre la base de: Ampliación de la producción, Mejora de la satisfacción del cliente, Retorno de la inversión garantizada; es así que en las instituciones de educación superior, las cuales tienen su esencia en la formación e investigación, se debe promover el aseguramiento de una mayor producción académica - científica que permita satisfacer al cliente en dos vertientes, la primera en función de brindar carreras pertinentes al buen vivir tanto en pregrado y posgrado, lo cual garantiza que los estudiantes puedan acceder a estudiar lo requerido según sus necesidades, perspectivas, pero sobre todo, lo requerido por el país para lograr contar con los profesionales capacitados para el abordaje integral del desarrollo socio económico.

Por otro lado, debe plantearse una mejora en la producción investigativa, mediante el fomento de investigaciones que permitan crear, innovar, tanto en ciencia, tecnología, 


\section{CIENCIAMATRIA}

Revista Interdisciplinaria de Humanidades, Educación, Ciencia y Tecnología

Año VI. Vol. VI. Nº10. Enero - Julio 2020

Hecho el depósito de ley: pp201602FA4721

ISSN-L: 2542-3029; ISSN: 2610-802X

Universidad Nacional Experimental Francisco de Miranda (UNEFM). Santa Ana de Coro. Venezuela

Alba Lucía Cáceres Larreátegui; Sandra Ximena Torres Mejía; Beatriz Jaqueline Briones Arteaga

como en la transformación comunitaria, mediante el abordaje de diversos paradigmas epistemológicos - metodológicos que permitan a los investigadores aportar a las demandas requeridas para la transformación social del Ecuador desde una perspectiva del buen vivir. Así mismo es pertinente enfocar la calidad de las producciones científicas, es decir, revistas y libros derivados de investigaciones que permitan ofrecer al lector, resultados y avances en consonancia a las exigencias actuales de la nación, con la finalidad de contar con datos para la generación de políticas y estrategias para el abordaje social desde el sector universitario.

Siendo pertinente trabajar en base a una gestión por procesos que permita la mejora continua como una referencia para el trabajo sistémico desde las instituciones de educación superior del Ecuador, así la ISO 9001: 2015, señala que propone un enfoque "basado en procesos", descrito como:

La comprensión y gestión de los procesos interrelacionados como un sistema contribuye a la eficacia y eficiencia de la organización en el logro de sus resultados previstos. Este enfoque permite a la organización controlar las interrelaciones e interdependencias entre los procesos del sistema, de modo que se pueda mejorar el desempeño global de la organización (ISO 9001: 2015, p. 8).

Esto implica actuar en la mejora de la totalidad de los procesos de las instituciones de educación superior, implica concatenar la docencia, investigación, abordaje comunitario, mediante una acción social donde se integren los actores que conforman la institución en un todo para trabajar desde las potencialidades que cada sector posee en pro de metas comunes, esto mediante el ciclo: Planificar, Hacer, Verificar, Actuar, siendo sus siglas "PHVA", la ISO 9001 (2015), plantea que es "un enfoque global de pensamiento basado en riesgos", así mismo, plantea que "la aplicación del enfoque a procesos en un sistema de gestión de la calidad permite:

a) La comprensión y el cumplimiento de los requisitos de manera coherente;

b) La consideración de los procesos en términos de valor agregado;

c) El logro de un desempeño del proceso eficaz;

d) La mejora de los procesos con base en la evaluación de los datos y la información (p. 8). 
El enfoque PHVA, podrá ser desarrollado en los procesos que conforman la institución de educación superior, por cuanto integra las potencialidades de todas las personas de la organización, siendo menester involucrar además los sub procesos elaborados por ella, en una conducción de ejecutar los planes operativos en donde se ven involucrados el liderazgo, planeación estratégica, toma de decisiones, presupuesto, entre otros elementos que se desarrollan en cada institución. La International Organization for Standarization 9001 (ISO) (2015), describe el ciclo PHVA:

1. Planificar: establecer los objetivos del sistema y sus procesos, y los recursos necesarios para generar y proporcionar resultados de acuerdo con los requisitos del cliente y las políticas de la organización, e identificar y abordar los riesgos y las oportunidades;

2. Hacer: implementar lo planificado;

3. Verificar: realizar el seguimiento y (cuando sea aplicable) la medición de los procesos y los productos y servicios resultantes respecto a las políticas, los objetivos, los requisitos y las-actividades planificadas, e informar sobre los resultados;

4. Actuar; tomar acciones para mejorar el desempeño, cuando sea necesario ( $p$. 10).

Desde la gestión por procesos, se busca brindar satisfacción al cliente en un entorno de calidad, esto implica que en relación a las instituciones de educación superior, el cliente es representado por la sociedad, por lo tanto, es menester trabajar desde un enfoque de calidad total que permita sobre la marcha del trabajo, evaluar y mejorar continuamente con la finalidad de construir una educación que contribuya a fortalecer las metas comunes planteadas desde una direccionalidad nacional, de ese modo, se articula una educación basada en la calidad.

La gestión por procesos permite adaptar la organización en funcionalidad del contexto donde se encuentra inmersa, Maya, Aldana Zavala \& Isea Argüelles (2019), plantean que "las posibilidades de mejora de las instituciones escolares, se basan en reconocer que estos procesos no pueden sólo prescribirse, por cuanto en ellos inciden factores tales como el compromiso, la iniciativa y la implicación, propios del contexto de cada 


\section{CIENCIAMATRIA}

Revista Interdisciplinaria de Humanidades, Educación, Ciencia y Tecnología

Año VI. Vol. VI. Nº10. Enero - Julio 2020

Hecho el depósito de ley: pp201602FA4721

ISSN-L: 2542-3029; ISSN: 2610-802X

Universidad Nacional Experimental Francisco de Miranda (UNEFM). Santa Ana de Coro. Venezuela

Alba Lucía Cáceres Larreátegui; Sandra Ximena Torres Mejía; Beatriz Jaqueline Briones Arteaga

institución" (p. 123). No solo basta con que las instituciones de educación superior, declaren en sus documentos doctrinales que trabajan sobre la base de una gestión de calidad, es necesario que los actores que la integran, se sientan comprometidos con trabajar para el logro de la misma.

Siendo necesario además que las instituciones de educación superior y el Estado, articulen la gestión de procesos para lograr satisfacer las demandas de la sociedad como cliente de la educación, tal como lo plantea Huapaya Capcha (2019), por consiguiente no puede considerarse exitoso los logros de una determinada institución educativa, sí estos se encuentran divorciados de la realidad social del entorno donde se encuentra, esto generaría un divorcio entre la gestión por procesos y el cliente (sociedad), siendo necesario tener en cuenta a Aldana, Salón \& Guzmán (2019), quienes explican que:

La sociedad globalizada de las dos últimas décadas ha conducido al mundo empresarial a reinventarse para poder ser competitivo, eficaz, productivo, ofrecer calidad a sus clientes. No obstante, en el contexto educativo por los nuevos paradigmas como la complejidad de Morin, entre otros, se encuentra en una metamorfosis donde debe empoderarse de nuevos estilos de aprendizaje, planificación, evaluación, investigación, gerencia, liderazgo, para estar en concordancia con los retos epistémicos de las instituciones postmodernas del conocimiento (p. 64).

La organización de la educación superior, debe estar en concordancia con las diversas exigencias empresariales, esto le permitirá conducirse en función de los lineamientos de calidad vigente, así mismo, desde lo curricular, se podrá auto evaluar como ejercicio para estar en consonancia con las tendencias educativas que procuran formar desde la integralidad del ser, siendo necesario brindar mayor apoyo a los estudiantes para que ingresen a la educación superior, tal como lo plantea LucasMuentes, Mendoza Andrade\& Lucas Muentes (2018).

Desde lo abordado, las instituciones de educación superior, al trabajar por gestión de procesos, pueden trabajar en la optimización de los procesos contables, administrativos que desarrollan, debido que al tener una planeación docente y de investigación, así como control del gasto presupuestario, podrán visualizar la eficacia de los procesos, siendo necesario que generen producción que les permita contar con 
recursos para fortalecer sus recursos financieros, siendo esto posible desde el enfoque por gestión.

\section{METODOLOGÍA DE LA INVESTIGACIÓN}

La investigación es de tipo correlacional, Chávez (2007), plantea que los "estudios correlaciónales tienen como propósito determinar el grado de relación que hay entre dos variables, detectando hasta qué punto las alteraciones de una, dependen de la otra" (p. 137), en este sentido, se midió la relación entre la gestión de la calidad y su incidencia administrativa financiera en las instituciones de Educación Superior del Ecuador, se abordó desde un diseño no experimental, transeccional de campo. Hernández, Fernández, Baptista (2014) refieren al diseño no experimental como "los estudios que se realizan sin la manipulación deliberada de variables y en los que sólo se observan los fenómenos en su ambiente natural para después analizarlos” (p. 267). Se abordó una población de 11 instituciones de educación superior a quienes se les aplicó un instrumento que permitió cotejar la gestión de calidad basada en procesos y lo relacionado a lo administrativo financiero.

\section{RESULTADOS}

Correlación de gestión de la calidad y administrativa financiera Cuadro 1

Gestión de calidad
Administrativa financiera

\begin{tabular}{llrr}
\hline G. C & Correlación de Pearson & 1 & 0,061 \\
& Sig. (bilateral) & & 0,434 \\
& N & 167 & 167 \\
Adm. F. & Correlación de Pearson & 0,063 & 1 \\
& Sig. (bilateral) & 0,434 & 167 \\
& N & 167 & 167 \\
\hline
\end{tabular}


La correlación de Pearson posee un resultado de 0,063, la cual se encuentra en el rango de correlación positiva media y una significancia de 0,434, acercándose a 0,5, generando un nivel de confianza positiva.

Se rechaza la hipótesis nula H0: la gestión de la calidad no se relaciona con la competencia administrativa financiera en las instituciones de Educación Superior del Ecuador. Aceptándose la hipótesis positiva $\mathrm{H} 1$ : la gestión de la calidad se relaciona con la competencia administrativa financiera en las instituciones de Educación Superior del Ecuador.

Se encontró una asociación lineal estadísticamente significativa, moderada ( $\mathrm{rP}=$ $0.434, p<0.05)$.

\section{Discusión}

Los resultados obtenidos denotan un coeficiente de Pearson de 0,061 , siendo una correlación positiva media en función de lo planteado por (Hernández, Fernández, Baptista, 2014, p. 305), esto implica que cuando se trabaja por gestión de procesos, se eleva la calidad de lo relacionado a la competencia administrativa financiera en las instituciones de Educación Superior del Ecuador correspondiéndose teóricamente con lo planteado por la International Organization for Standarization 9001 (ISO) (2015), en cuanto al ciclo PHVA,

1. Planificar: establecer los objetivos del sistema y sus procesos, y los recursos necesarios para generar y proporcionar resultados de acuerdo con los requisitos del cliente y las políticas de la organización, e identificar y abordar los riesgos y las oportunidades;

2. Hacer: implementar lo planificado;

3. Verificar: realizar el seguimiento y (cuando sea aplicable) la medición de los procesos y los productos y servicios resultantes respecto a las políticas, los objetivos, los requisitos y las-actividades planificadas, e informar sobre los resultados;

4. Actuar; tomar acciones para mejorar el desempeño, cuando sea necesario ( $p$. 10). 


\section{CONCLUSIONES}

Desde los resultados planteados, se cumple con el propósito establecido por la gestión de procesos, lo cual procura optimizar los sub procesos que conforman la organización en funcionalidad de articular dimensiones integradoras de las expectativas sociales en torno a la educación superior del Ecuador, lo cual implica concebir la educación como un proceso de mejora continua, donde intervienen no solo lo relacionado al aprendizaje, sino que al hablar de instituciones de educación superior, se genera un andamiaje empresarial que debe redundar en beneficios para la sociedad.

Por lo tanto la universidad no puede ser percibida como un ente meramente reproductor de profesionales, sino, como una alianza en favor de promover transformaciones desde su quehacer formativo, siendo necesario articular docencia, investigación, trabajo administrativo y gerencial, como un proceso que permite conciliar en favor de brindar respuestas conducentes a la conformación de una educación de calidad.

En este sentido, educación de calidad engloba diversos factores que deben ser tenidos en cuenta para la conformación de una gestión basada en lo sistémico como alternativa para consolidar una visión organizativa acorde a los retos globales, lo cual implica formar desde lo glocal para el mundo, siendo pertinente fomentar competencias en los actores que conforman las instituciones con el favor de promover el compromiso, responsabilidad, como factores importantes de la identidad corporativa en función de trabajar sinérgicamente en el logro de metas comunes que satisfagan las demandas del cliente (sociedad).

\section{REFERENCIAS CONSULTADAS}

1. Aldana, J., Salón, M., \& Guzmán, N. (2019). Liderazgo sistémico en las competencias gerenciales docentes universitarias. CIENCIAMATRIA, 5(8), 5074. Recuperado a partir de http://cienciamatriarevista.org.ve/index.php/cm/article/view/87

2. Chávez, N. (2007). Introducción a la Investigación Educativa. Maracaibo, Venezuela: Ediciones Gráfica, C.A. 
3. Hernández, R., Fernández, C., Baptista, P. (2014). Metodología de la Investigación. México: Editorial Mc - Graw - Hill Interamericana.

4. Huapaya Capcha, Y. (2019). Gestión por procesos hacia la calidad educativa en el Perú. Revista Arbitrada Interdisciplinaria Koinonía, 4(8), 243-261. doi:http://dx.doi.org/10.35381/r.k.v4i8.277

5. Lucas-Muentes, A., Mendoza Andrade, M., \& Lucas Muentes, J. (2018). La calidad de la educación superior: referentes para su desarrollo en contexto Ecuatoriano. Polo del Conocimiento, 3(3), 37-52. doi:http://dx.doi.org/10.23857/pc.v3i3.443

6. Maya, E., Aldana Zavala, J., \& Isea Argüelles, J. (2019). Liderazgo Directivo y Educación de Calidad. CIENCIAMATRIA, 5(9), 114 - 129. https://doi.org/10.35381/cm.v5i9.102

7. Organización Internacional de Normalización (2015). ISO 9001: 2015. Recuperado de http://sigug.uniguajira.edu.co:8080/sigug/pdf/ISO\%209001\%202008.pdf

\section{REFERENCES CONSULTED}

1. Aldana, J., Salón, M., \& Guzmán, N. (2019). Systemic leadership in university teaching management skills. SCIENCE, 5 (8), 50-74. Recovered from http://cienciamatriarevista.org.ve/index.php/cm/article/view/87

2. Chavez, N. (2007). Introduction to educative research. Maracaibo, Venezuela: Ediciones Gráfica, C.A.

3. Hernández, R., Fernández, C., Baptista, P. (2014). Investigation methodology. Mexico: Editorial Mc - Graw - Interamerican Hill.

4. Huapaya Capcha, Y. (2019). Management by processes towards educational quality in Peru. Interdisciplinary Arbitrated Review Koinonía, 4 (8), 243-261. doi: http: //dx.doi.org/10.35381/r.k.v4i8.277

5. Lucas-Muentes, A., Mendoza Andrade, M., \& Lucas Muentes, J. (2018). The quality of higher education: references for its development in Ecuadorian context. Pole of Knowledge, 3 (3), 37-52. doi: http:

//dx.doi.org/10.23857/pc.v3i3.443

6. Maya, E., Aldana Zavala, J., \& Isea Argüelles, J. (2019). Leadership Leadership and Quality Education. CIENCIAMATRIA, 5 (9), 114-129. Https://doi.org/10.35381/cm.v5i9.102 


\section{CIENCIAMATRIA}

Revista Interdisciplinaria de Humanidades, Educación, Ciencia y Tecnología

Año VI. Vol. VI. N¹0. Enero - Julio 2020

Hecho el depósito de ley: pp201602FA4721

ISSN-L: 2542-3029; ISSN: 2610-802X

Universidad Nacional Experimental Francisco de Miranda (UNEFM). Santa Ana de Coro. Venezuela

Alba Lucía Cáceres Larreátegui; Sandra Ximena Torres Mejía; Beatriz Jaqueline Briones Arteaga

7. International Organization for Standardization (2015). ISO 9001: 2015.

Retrieved from

http://sigug.uniguajira.edu.co:8080/sigug/pdf/ISO\%209001\%202008.pdf

(C2020 por los autores. Este artículo es de acceso abierto y distribuido según los términos y condiciones de la licencia Creative Commons Atribución-NoComercial-Compartirlgual 4.0 Internacional (CC BY-NC-SA 4.0) (https://creativecommons.org/licenses/by-nc-sa/4.0/). 